Review

\title{
Brodie's Abscess: A Systematic Review of Reported Cases
}

\author{
Niels van der Naald ${ }^{1,2}$, Diederik P.J. Smeeing1,2, Roderick M. Houwert ${ }^{2,3}$, Falco Hietbrink2,3, Geertje A.M. \\ Govaert ${ }^{2,3}$, Detlef van der Velde ${ }^{1}$ \\ 1. St Antonius Hospital Nieuwegein, Department of Surgery, The Netherlands \\ 2. University Medical Center Utrecht, Department of Surgery, The Netherlands \\ 3. Traumacenter Utrecht, The Netherlands \\ $\triangle$ Corresponding author: Niels van der Naald, University Medical Center Utrecht, 3584 CX Utrecht, The Netherlands. Phone: +3188 7555555; E-mail: \\ n.vandernaald@umcutrecht.nl \\ (C) Ivyspring International Publisher. This is an open access article distributed under the terms of the Creative Commons Attribution (CC BY-NC) license \\ (https://creativecommons.org/licenses/by-nc/4.0/). See http://ivyspring.com/terms for full terms and conditions.
}

Received: 2018.11.28; Accepted: 2018.12.28; Published: 2019.01.24

\begin{abstract}
Introduction: Brodie's abscess is a form of osteomyelitis. Since its first appearance in the medical literature in 1832, numerous cases have been described. The aim of this article is to provide the first comprehensive overview of published cases of Brodie's abscess, and to describe diagnostic methods, therapeutic consequences and outcomes.

Methods: According to PRISMA guidelines a systematic review of the literature was performed. All published data in English or Dutch were considered for inclusion with no limitations on publication date. Data was extracted on demography, duration of symptoms, signs of inflammation, diagnostic imaging, causative agent, treatment and follow-up.

Results: A total of 70 articles were included, reporting on a total of 407 patients, mostly young (median age 17) males (male:female ratio 2.1:1). The median duration of symptoms before diagnosis was 12 weeks (SD 26). Mostly consisting of pain (98\%) and/or swelling (53\%). 84\% of all patients were afebrile, and less than $50 \%$ had elevated serum inflammation markers. Diagnosis was made with a combination of imaging modalities: plain X-ray in 96\%, MRI (16\%) and CT-scan (8\%). Treatment consisted of surgery in $94 \%$ of the cases, in conjunction with long term antibiotics in $77 \%$. Staphylococcus aureus was the pathogen most often found in the culture $(67,3 \%)$. Outcome was generally reported as favorable. Recurrence was reported in $15,6 \%$ of the cases requiring further intervention. Two cases developed permanent disability.

Conclusion: Brodie's abscess has an insidious onset as systemic inflammatory signs and symptoms were often not found. Treatment consisted mostly of surgery followed by antibiotics $(77 \%)$ or only surgery $(17 \%)$ and outcomes were generally reported as favourable.
\end{abstract}

Key words: Brodie's abscess, osteomyelitis, case report, systematic review

\section{Introduction}

Brodie's abscess is a sub-acute form of osteomyelitis, presenting as a collection of pus in bone, often with an insidious onset(1). It's first description in the medical literature was in 1832 by sir Benjamin Brodie during a lecture held for the Medical and Surgical Society(2). The first case he described, a 24-year old male, had suffered half his life with bouts of severe pain originating from a bony swelling above the right ankle. Since no other treatment was successful they decided to amputate the affected limb. Examination of the amputated specimen showed a walnut sized collection of pus. This is when Brodie hypothesized that limb sparing surgery by means of trephination and thus drainage of the abscess should 
be the cornerstone of treatment. In this pre-antibiotic era this was the only treatment option available. There are several etiologies concerning bone infections: Fracture related bone infection after (open) trauma(3)(4), exposed bone in diabetic wounds or haematogenous osteomyelitis $(5,6)$. The primary site of bacterial spread is often unknown. It is hypothesized that susceptibility of bone is increased after bone bruising resulting from minor prior trauma without open wounds or a fracture. Brodie's abscess is a contained infection in the myelum, surrounded by a sclerotic wall. Thus leading to little systematic inflammatory response.

Other diseases can present similarly to Brodie's abscess and warrant further investigations, such as osteoid osteoma, Langerhans cell histiocytosis, chondrasarcoma, eosinphilic granuloma or tuberculosis (7-11).

Treatment of a Brodie's abscess is based on the preference of the health care professional, as no publications other than case reports and series are available. The aims of this article are description of the clinical characteristics, surgical treatment, outcome and consequence of Brodie's abscess based on the case-reports described in the medical literature up to now.

\section{Methods}

The study was registered in the PROSPERO database with registration number CRD42018093918. A priori to execution of this study, the search strategy and data extraction procedures were defined. No amendments were made during study execution. We defined Brodie's abscess as a collection of pus in bone after suspected haematogenous spread of bacteria, without a history of open trauma or surgery.

\section{Search strategy and selection criteria}

This review was performed according to the Preferred Reporting Items for Systematic reviews and Meta-Analyses (PRISMA) guidelines(12). Articles concerning a Brodie's abscess in patients of all ages were included. Eligibility criteria for inclusion of articles were defined a priori. Two reviewers (NN and DS) independently conducted an electronic systematic search in PubMed/MEDLINE, Embase, Cochrane Central Register of Controlled Trials (CENTRAL from The Cochrane Library) and CINAHL for articles published up to April 12 ${ }^{\text {th }} 2018$. The search syntax is provided in Figure 1. A clinical librarian was consulted on the search strategy. Titles and abstracts of retrieved citations were screened, and both reviewers read the full text of potentially suitable studies. Articles were included if written in English or Dutch language. Case reports have also been included. Studies were excluded if they concerned bone infections after surgery, open trauma or caused by Mycobacterium tuberculosis. Only published data have been included. Comments, abstracts for conferences, study protocols, reviews, biomechanical studies and animal studies were excluded. No further search restrictions or search filters were applied in the search. Disagreements in the search were resolved by discussion with a third, independent reviewer (DV).

Searchstring PubMed/MEDLINE (n=172) at $\mathbf{1 2}^{\text {th }}$ April 2018
(Brodie[Title/Abstract] OR Brody[Title/Abstract] OR
Brodie's[Title/Abstract] OR Brody's[Title/Abstract]) AND
(abscess*[Title/Abstract] OR abscess[MeSH Terms])
Searchstring Embase ( $\mathbf{n}=193$ ) at $12^{\text {th }}$ April 2018
(Brodie:ti,ab OR Brody:ti,ab OR 'Brodie/s':ti,ab OR
'Brody/s':ti,ab) AND (abscess*:ti,ab OR 'abscess'/exp)
Searchstring CENTRAL (n=49) at 12 ${ }^{\text {th }}$ April 2018
Brodie OR abscess
Searchstring CINAHL (n=11) at $12^{\text {th }}$ April 2018
(abscess OR abscess $)$ AND (Brodie OR Brody OR Brodie's
OR Brody's)

Figure 1. Search syntax

\section{Quality assessment}

No published, peer reviewed and validated quality assessment tool for case reports or case series is currently available. Therefore quality assessment was not performed.

\section{Data extraction}

The following data were extracted: first author, year of publication, country in which the study is performed, number of included patients, age, sex, presenting complaints and duration, body temperature, findings on physical examination, serum inflammatory markers erythrocyte sedimentation rate (ESR), White Blood Count (WBC) and C-reactive protein (CRP), location of Brodie's abscess, diagnostic tools (including imaging and cultures), treatment and outcome at follow-up if reported.

\section{Data analysis}

Descriptive statistics were used to display the means, standard deviations and ranges for all outcome measures as described in the included studies. Dichotomous data were presented as frequencies with percentages or as proportions. Data were converted into the same units if needed. If a study had missing data, available case analysis was performed. 


\section{Results}

\section{Search}

The electronic searches detected 425 articles. These papers were screened on title and abstract. In total, 70 articles could be included. The search results, reasons for exclusion and selection process is summarized in the flowchart in Figure 2. All included articles are referenced in Appendix 1.
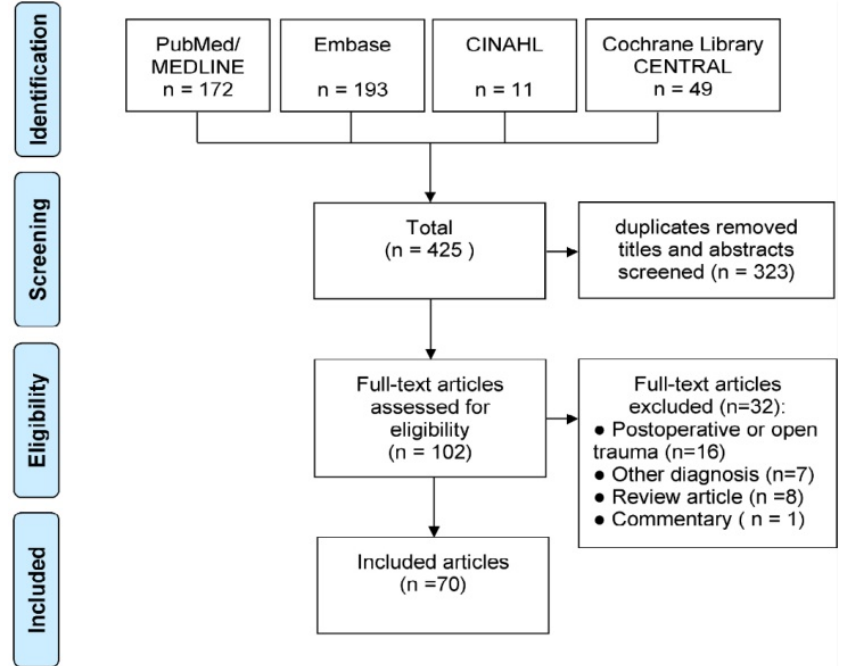

Figure 2. PRISMA flow chart

\section{Baseline characteristics}

A total of 70 studies were included dated from 1921 to 2018 including a total of 407 patients. Publications originated from all over the world, mostly from the United States of America $(n=16)$, the United Kingdom $(n=10)$ and India $(n=6)$. The median age was 17 years (SD11) $(n=169 ;$ Boriani(13) and Gillespie(14) did not report on age). Brodie's abscess was more common in men (men $n=274$; female $n=131$ ) with a male:female ratio 2.1:1. (Bogoch(15), Strobel(16) and Tan(17) did not report the male:female ratio $\mathrm{n}=8$ ). A complete overview of the baseline characteristics is presented in Appendix 1.

\section{Findings}

The included studies had a large heterogeneity on reported items. Listed below are the main findings of cases that reported on that specific item. The location of Brodie's abscesses was reported to be distributed all over the body, as presented in figure 3 . In those cases describing laterality, the right side was more often involved than the left, with 68/115 (59\%) cases. The median duration of complaints before the diagnosis Brodies abscess was established was 12 weeks (SD 26). In 56 cases a history concerning possible pathogenesis was noted. A history of recent infection was positive in $12 / 56$ cases, with the location of this infection only described in three cases: all respiratory infections in children $<12$ years old. A history of (minor) trauma without fracture or open wound to the effected limb was described in 25/56 cases. Pain was most frequently described as presenting complaint, in 141/144 (98\%), accompanied by swelling in 76/144 (53\%) of the cases. Only three times no pain was reported. Once with just a swelling, twice with a draining sinus tract. In 82/98 of those cases reporting on temperature the patient was afebrile. Serum inflammation markers were mostly normal or slightly elevated: Erythrocyte Sedimentation Rate $<20 \mathrm{~mm} / \mathrm{hr}$ in 51/92, White Blood Cells count $<10 \times 10^{9} / \mathrm{L}$ in $61 / 87$ and $<10 \mathrm{mg} / \mathrm{dL}$ C-Reactive Protein in 31/40 of the cases. Diagnostic imaging consisted of x-ray in 335/350 (96\%) cases, most often combined with magnetic resonance imaging (MRI) $57 / 350(16 \%)$ and/or computed tomography (CT) in $28 / 350(8 \%)$ cases. Other modalities were ultrasound and nuclear imaging techniques. Staphylococcus aureus was the most frequently found pathogen if cultures were taken and reported on: 178/264 (67\%) cases. Of these 6 were reported as Methicillin-resistant Staphylococcus aureus (MRSA). No growth was reported in $68 / 264(25.8 \%)$ of the cases. The outcome of all cultures is presented in Figure 4. Treatment mostly consisted of surgery (incision and drainage with or without direct closure and drain, curettage, percutaneous bone biopsy/washout) and a course of antibiotics (spanning from 10 days to more than three months) in $163 / 212(77 \%)$ cases, surgery alone was mentioned in $37 / 212(17 \%)$ of the cases, and only antibiotic treatment in $11 / 212(5 \%)$ cases. A total number of $128 / 407(31,5 \%)$ cases mentioned both length of follow-up and results (median 32.7 months, SD 57). Of these, $106 / 128$ (83\%) reported no recurrence, $20 / 128(15,6 \%)$ reported recurrence with need of re-operation and/or antibiotic therapy and only two cases had ongoing complaints (1x neurologic deficits due to a collapsed vertebra and $1 x$ leg length discrepancy).

\section{Discussion}

This paper presents a systematic review of reported Brodie's abscess. To the authors knowledge this is the first systematic review and comprehensive summary of all published cases concerning this disease. We found that Brodie's abscess was most frequently encountered in young men. Brodie's abscess has been described as a subacute presentation of osteomyelitis (1). We have found a median of 12 weeks of complaints before diagnosis was established. In line with the original case series presented by Brodie, the tibia (48.6\%) and femur (31.1\%) were mostly involved. Only 56 cases clearly reported on a 
possible etiology, with both recent infection and (minor) trauma without fracture being present.

The limited benefit of serum inflammation markers in fracture related infections has been studied before (18) and our findings in Brodie's abscess are comparable.

Many imaging techniques have been described to diagnose Brodie's abscess; conventional $x$-ray, CT-scan, MRI and nuclear medicine imaging modalities. In children, the modality of choice to distinguish between infection and bone tumors is MRI (19). Magnetic resonance imaging has also been proven to be superior then plain x-ray in adults to diagnose osteomyelitis (20,21). Recent studies also show that scintigraphy with SPECT/CT or FDG-PET combined with CT give great diagnostic accuracy in haematogenous osteomyelitis and for fracture related infections, but these papers do not specifically address Brodie's Abscess (22,23). Unfortunately, these techniques are also not readily available in many hospitals, especially not in the geographic areas with higher incidence of osteomyelitis. Therefore conventional radiographs are still the most utilized diagnostic imaging modality for most physicians when considering the diagnosis of Brodie's abscess. In line with previous reports $(18,19)$ Staphylococcus aureus is the dominant pathogen found. Only in six cases culture showed MRSA to be present. We hypothesize that this is an underrepresentation due to inadequate testing or reporting. Salmonella spp. were found in 4 cases, all adults. Since its first reported link to osteomyelitis in 1982(24), Kingella kingae has been found in 3 cases. It has been suggested that $K$. kingae might be responsible for more bone and joint infection then was previously known because of improved diagnostic techniques such as polymerase chain reaction (PCR)(25). There is also gathering evidence that faster diagnosis of bone infection is possible through these techniques $(26,27,28)$.

Treatment consisted primarily of surgery $(94 \%)$ often in combination with antibiotics (77\%). Only 11 cases were treated solely with antibiotics. Overall, outcome was poorly documented. Less than $1 / 3$ rd of the cases reported both on length of follow-up and the means used. Of those cases, $15 \%$ reported a recurrence, lower than the $20 \%$ recurrence found for chronic osteomyelitis (29).

When interpreting our findings, the authors believe that surgery is still the cornerstone of treatment. After drainage and obtaining material for culture and cytology/histology, antibiotics should be administered. The length of antibiotic treatment is unclear.

Incidence of osteomyelitis is unevenly distributed in the world, with developing countries bearing the brunt (30). Publications are dominantly from developed countries and could therefore show a distorted picture. Olasinde et al (31) $(\mathrm{N}=20)$ reports the only series from a developing country, Nigeria. Another 6 case reports are from India. This most likely results in a publication bias.

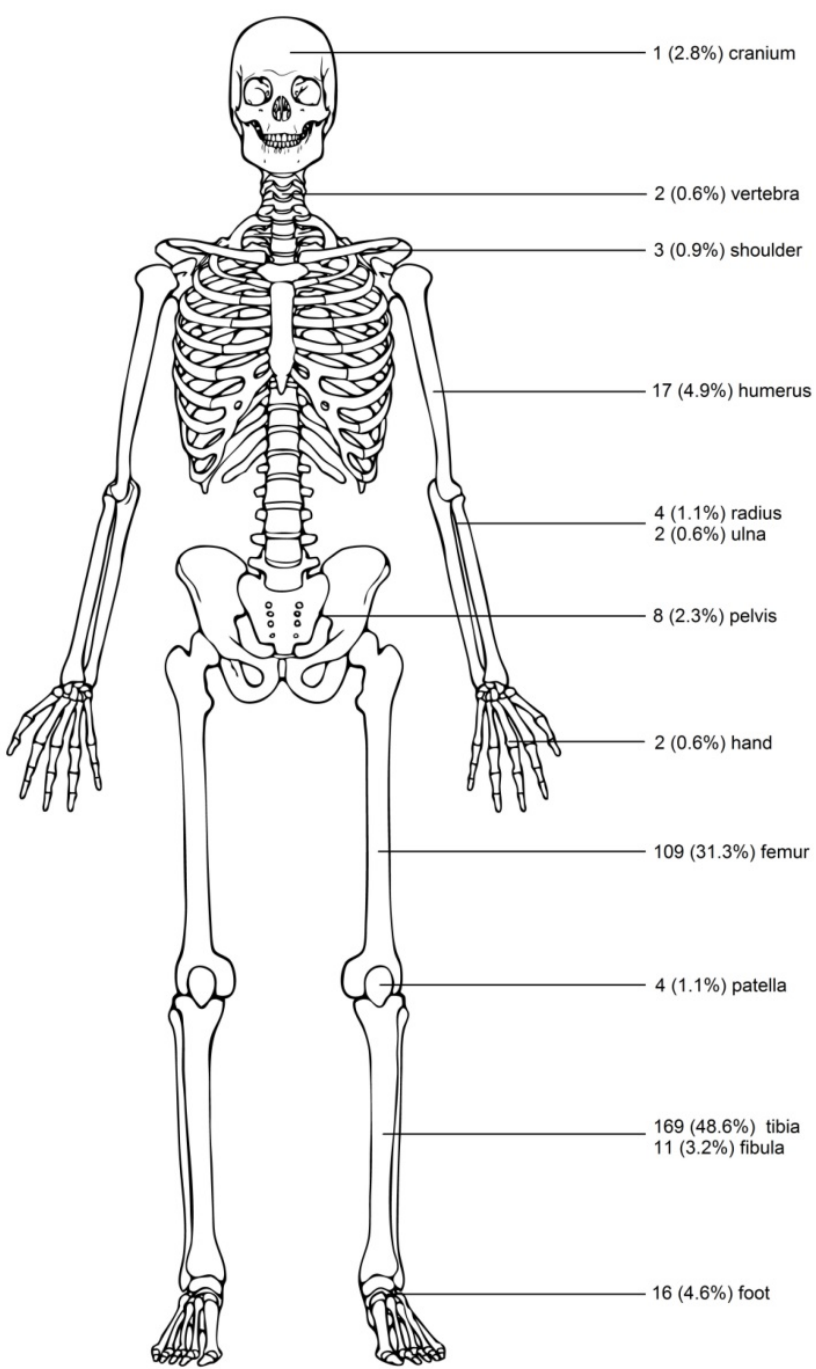

Figure 3. Anatomical distribution of Brodie's abscess in the included literature

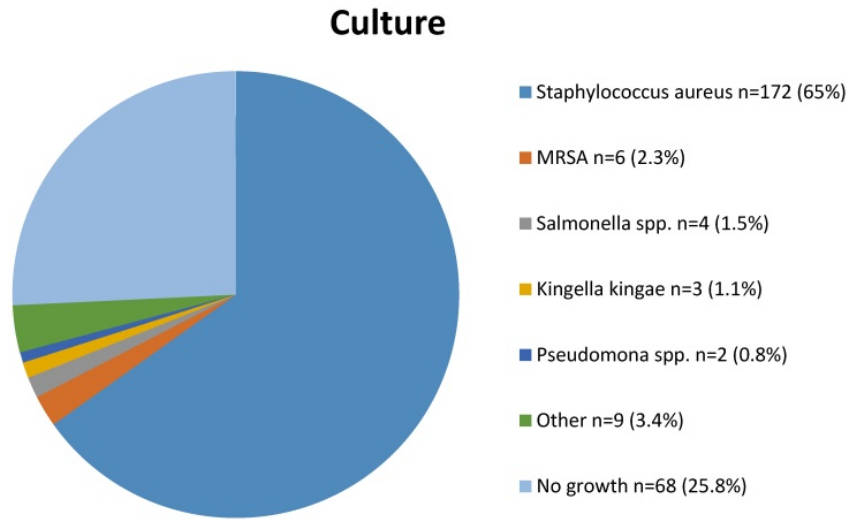

Figure 4. Reported microbiological results of cultures taken from Brodie's abscess in the included literature 
The studies of Boriani(13) $(\mathrm{n}=181)$ and Gillespie et al (14) $(\mathrm{N}=57)$ are the largest series and therefore dominant in the outcome of our study. Unfortunately, they also gave little detail about the presentation of the patient, causative bacteria, diagnostics, treatment and effect. Case reports are mostly used to illustrate a rare disease or a rare presentation of a more common disease. Thus it is likely that non-traditional presentations are over-reported, something that large series would offset. Cases that clearly followed from non-haematogenous types of bone infection (such as open fractures or after fracture surgery) were excluded. Not all authors reported in depth on the history of their patient, especially in the larger series history this was not included. Eligibility for inclusion was based on the authors of the case report to diagnose Brodie's abscess following imaging and surgery. No selection protocol was used for the precise infection diagnosis.

Outcomes were poorly reported on in the larger series. In the cases that did mention outcome, the length of follow-up was either not given or a large variability in length and means of follow-up was present.
Because of the decrease in incidence in developed countries we have sought to limit our exclusion criteria. We have not restricted our search by publication date and have included all languages that the authors could read sufficiently. This is a descriptive study, not measuring an outcome. We were limited to case reports and case series, all observational and with large heterogeneity on reported items. A quality assessment tool for case reports and series has, to our knowledge, not been peer reviewed and published.

Two clinical examples of Brodies abcesses are presented in Figure 5 and 6.

\section{Conclusion}

Brodie's abscess has an insidious onset and mainly presents with localized tenderness, but with absence of systemic inflammatory signs and symptoms. Diagnosis can be made with a number of imaging techniques ( $\mathrm{x}$-ray, CT, MRI). The tibia and femur are mostly affected. Surgical debridement is the cornerstone of treatment, often in conjunction with antibiotics. Mostly good outcomes are reported.
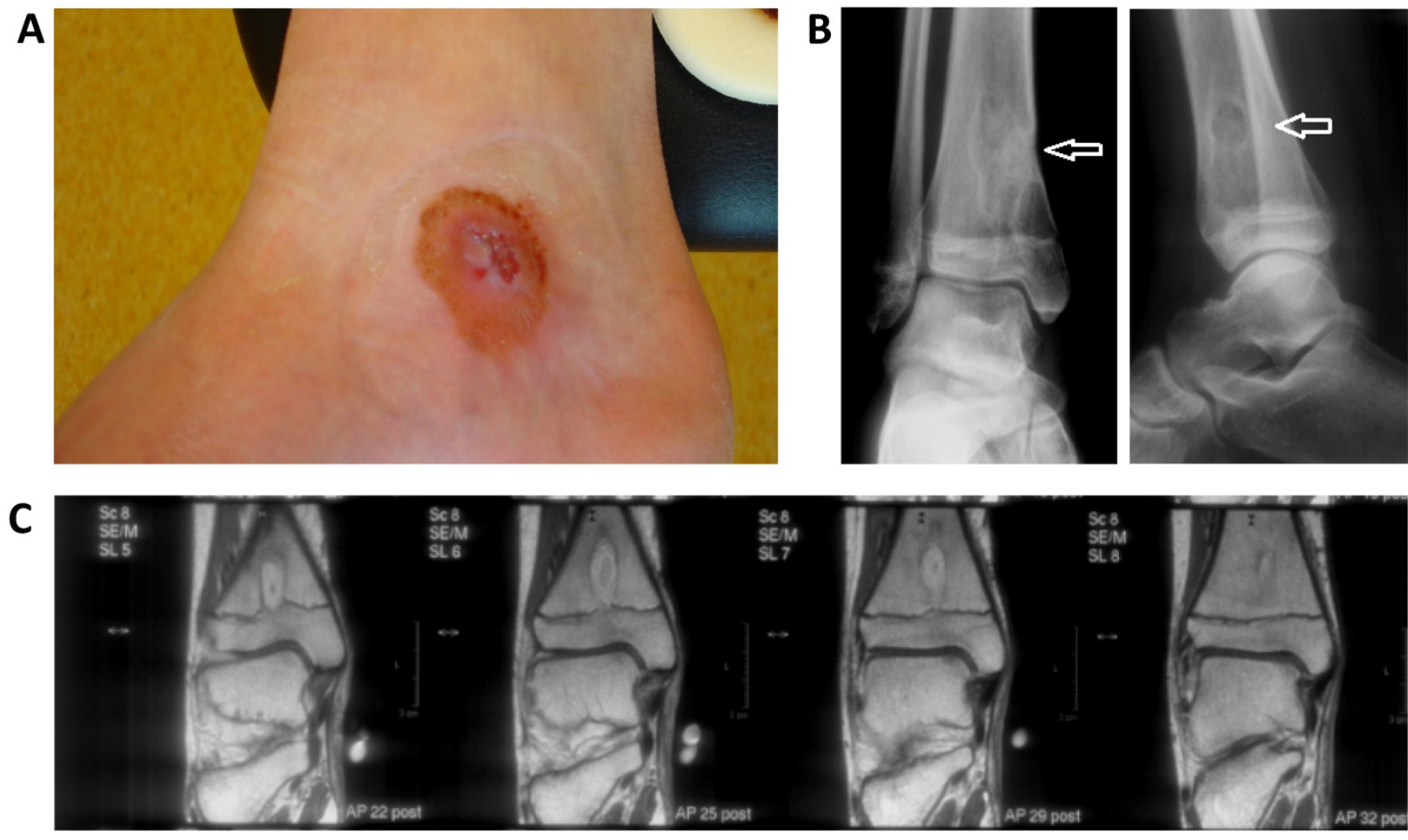

Figure 5. Case 1: A 14-year old girl with no prior medical or surgical history presented with a chronic wound. She strained her right ankle 6 months prior, with a non-revealing x-ray of the ankle taken at the local clinic. Previous treatment of the wound by other health care specialists included antibiotics and wound dressings. On presentation in our clinic a draining sinus tract is seen. Conventional x-ray showed a bone lesion that prompted further imaging with a MRI scan. In the metaphysis of the distal tibia a cystic lesion with rim enhancement is seen, suggestive of a Brodie's abscess. Surgical debridement was performed followed by insertion of gentamycin beads and primary closure. Ten days later these were removed and the defect was filled with autologous bone graft. Pus samples sent for microbiology examination revealed staphyloccus aureus. No further course of antibiotics were prescribed. During the 10 year follow-up she was free from complaints. (A) Draining sinus on the medial side of the right ankle in a 14-year old girl. (B) AP and Lateral x-ray of the right ankle of a 14-year old girl. Arrow pointing at a lytic lesion in de distal tibia. (C) MRI with coronal views of the right ankle with Gadolinium of a 14-year old girl. Bone lesion with rim enhancement suggestive of a Brodie's abscess in the metaphysis of the distal tibia. 
A
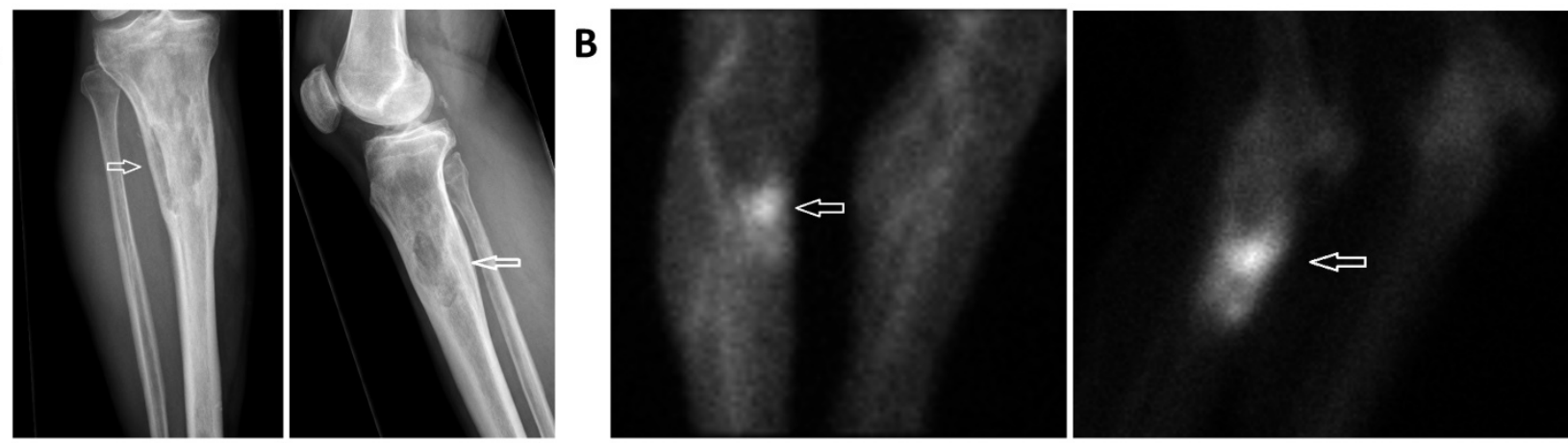

C
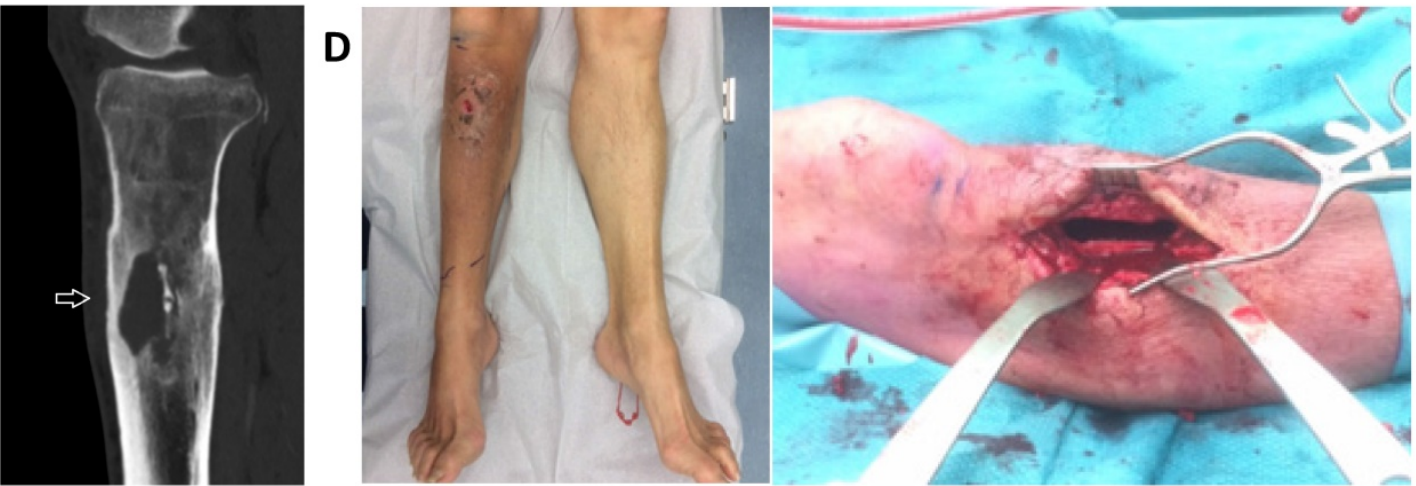

Figure 6. Case 2: A 68-year old man with a history of atherosclerotic disease presented at the clinic with recurrent pain below the right knee. During these pains he was unable to weight bear, but normally a short course of NSAID would quickly help him back on his feet. This time the pain persisted. Clinical examination showed a tender, non-fluctuating swelling over the right prox tibia. Serum inflammatory markers were within normal range but the $x$ ray showed cortical widening of the proximal tibia and sclerotic changes in the medulla. A scintigraphy showed increase uptake in all three phases. A CT scan showed a radiolucent lesion in the proximal metaphysis of the tibia of $2.5 \times 2.6 \times 3.5 \mathrm{~cm}$. There was sclerotic thickening of the cortex and a sinus to the anterior tibia with a small sequestrum seen centrally. Treatment followed with sequestrectomy and surgical debridement of the abscess and sinus tract. He received oral clindamycin for 1 week and was allowed to bear weight directly. Cultures taking peroperatively came back positive for staphylococci aureus. (A) AP and lateral x-ray of the right proximal tibia of a 68-year old man. Arrow pointing at lytic lesion. (B) Early and late phase bone scintigraphy of the lower extremities of a 68-year old man. Arrow pointing at heightened uptake located at the right proximal tibia. (C) Corronal CT image of right tibia of a 68-year old man. Arrow pointing at cystic lesion with sclerotic thickening of the cortex. (D) Pre-operative photo of a 68-year old man, showing progression of local inflammation around the right proximal tibia. Peri-operative photo of same patient showing bone window and debridement of Brodie's Abscess.

\section{Supplementary Material}

Appendix 1. http://www.jbji.net/v04p0033s1.pdf

\section{Acknowledgments}

All authors declare that they did not receive any kind of funding to write this article.

\section{Author contribution}

NvdN and DS were responsible for the study design, performed the literature search and interpreted the data. DvdV and GG were responsible for the study design and supervision of its execution. $\mathrm{RH}$ and FH supervised with the scientific writing and attributed their experience with surgical treatment of infectious diseases. The final manuscript has been approved by all authors.

\section{Competing Interests}

The authors have declared that no competing interest exists.

\section{References}

1. Harris NH, Kirkaldy WH. Primary Subacute Pyogenic osteomyelitis. J Bone Joint Surg Br. England; 1965 Aug;47:526-32.

2. Brodie BC. An Account of some Cases of Chronic Abscess of the Tibia. Med Chir Trans. 1832;17:239-49.

3. Metsemakers WJ, Morgenstern M, McNally MA, Moriarty TF, McFadyen I, Scarborough M, et al. Fracture-related infection: A consensus on definition from an international expert group. Injury. Netherlands; 2018 Mar;49(3):505-10.

4. Peng J, Ren Y, He W, Li Z, Yang J, Liu Y, et al. Epidemiological, Clinical and Microbiological Characteristics of Patients with Post-Traumatic Osteomyelitis of Limb Fractures in Southwest China: A Hospital-Based Study. J bone Jt Infect. Australia; 2017;2(3):149-53.

5. Lew DP, Waldvogel FA. Osteomyelitis. Lancet. 2004;369-79.

6. Hotchen AJ, McNally MA, Sendi P. The Classification of Long Bone Osteomyelitis: A Systemic Review of the Literature. J bone Jt Infect. Australia; 2017;2(4):167-74.

7. Schlur C, Bachy M, Wajfisz A, Ducou Le Pointe H, Josset P, Vialle R. Osteoid osteoma mimicking Brodie's abscess in a 13-year-old girl. Pediatr Int. 2013;55(2):29-31.

8. Chang W, Hsu Y, Wu Y, Kuo C, Huang G. Localized Langerhans Cell Histiocytosis Masquerading as Brodie's Abscess in a 2-year Old Child: a Case Report. EXCLI J. 2016;15:33-7.

9. Datir A, Lidder S, Pollock R, Tirabosco R, Saifuddin A. High-grade chondrosarcoma mimicking Brodie's abscess. Clin Radiol. The Royal College of Radiologists; 2009;64(9):944-7.

10. Siddiqui MS, Javed S, Razak a, Zubairy a, Khan SH. Brodie 'S Abscess With Tuberculous Osteomyelitis of the Foot. JBR-BTR J Belge Radiol. 2014;97:168-9.

11. Yoshikawa M, Sugawara Y, Kikuchi T, Nakata S, Mochizuki T, Ikezoe J, et al. Two cases of pediatric bone disease (Eosinophilic Granuloma and Brodie's abscess) showing similar scintigraphic and radiographic findings. Clin Nucl Med. 2000 Dec;25(12):986-90. 
12. Moher D, Liberati A, Tetzlaff J, Altman DG. Preferred reporting items for systematic reviews and meta-analyses: the PRISMA statement. BMJ. England; 2009 Jul;339:b2535.

13. Boriani S. Brodie's abscess. A study of 181 cases, with special reference to radiographic diagnostic criteria. Ital J Orthop Traumatol. 1981 Dec;6(3):373-83.

14. Gillespie WJ, Moore TE, Mayo KM. Subacute pyogenic osteomyelitis. Orthopedics. 1986 Nov;9(11):1565-70.

15. Bogoch E, Thompson G, Salter RB. Foci of chronic circumscribed osteomyelitis (Brodie's abscess) that traverse the epiphyseal plate. J Pediatr Orthop. 1984 Mar;4(2):162-9.

16. Strobel K, Hany TF, Exner GU. PET/CT of a brodie abscess. Clin Nucl Med. United States; 2006 Apr;31(4):210.

17. Tan K, Yoong P, Marshall TJ, Martin C. Percutaneous drainage as a novel approach for the treatment of Brodie's abscess. Clin Radiol. 2012 Oct; $67(10): 1030-3$

18. Bosch P, van den Kieboom J, Plate JDJ, IJpma FFA, Houwert RM, Huisman A, et al. Limited Predictive Value of Serum Inflammatory Markers for Diagnosing Fracture-Related Infections: results of a large retrospective multicenter cohort study. J bone Jt Infect. Australia; 2018;3(3):130-7.

19. Jaramillo D, Dormans JP, Delgado J, Laor T, St Geme JW. Hematogenous Osteomyelitis in Infants and Children: Imaging of a Changing Disease. Radiology. 2017;283(3):629-43.

20. Erdman WA, Tamburro F, Jayson HT, Weatherall PT, Ferry KB, Peshock RM. Osteomyelitis: characteristics and pitfalls of diagnosis with MR imaging. Radiology. United States; 1991 Aug;180(2):533-9.

21. Morrison WB, Schweitzer ME, Bock GW, Mitchell DG, Hume EL, Pathria MN, et al. Diagnosis of osteomyelitis: utility of fat-suppressed contrast-enhanced MR imaging. Radiology. United States; 1993 Oct;189(1):251-7.

22. Govaert GA, IJpma FF, McNally M, McNally E, Reininga IH, Glaudemans AW. Accuracy of diagnostic imaging modalities for peripheral post-traumatic osteomyelitis - a systematic review of the recent literature. Eur J Nucl Med Mol Imaging. 2017;44(8):1393-407.

23. Termaat MF, Raijmakers PGHM, Scholten HJ, Bakker FC, Patka P, Haarman HJTM. The accuracy of diagnostic imaging for the assessment of chronic osteomyelitis: a systematic review and meta-analysis. J Bone Joint Surg Am. United States; 2005 Nov;87(11):2464-71.

24. Davis JM, Peel MM. Osteomyelitis and septic arthritis caused by Kingella kingae. J Clin Pathol. 1982;35(2):219-22.

25. Slinger R, Moldovan I, Bowes J, Chan F, Slinger R, Moldovan I, et al. Polymerase chain reaction detection of Kingella kingae in children with culture-negative septic arthritis in eastern Ontario. Paediatr Child Health. 2016;21(2):79-82

26. Lausmann C, Zahar A, Citak M, Branes J, Schmidl S, Frommelt L, et al. Are There Benefits In Early Diagnosis Of Prosthetic Joint Infection With Multiplex Polymerase Chain Reaction? J bone Jt Infect. Australia; 2017;2(4):175-83.

27. Gallazzi E, Drago L, Baldini A, Stockley I, George DA, Scarponi S, et al. "Combined Diagnostic Tool" APPlication to a Retrospective Series of Patients Undergoing Total Joint Revision Surgery. J bone Jt Infect. Australia; 2017;2(2):107-13

28. Riccio G, Cavagnaro L, Akkouche W, Carrega G, Felli L, Burastero G. Qualitative Alpha-defensin Versus The Main Available Tests For The Diagnosis Of Periprosthetic Joint Infection: Best Predictor Test? J bone Jt Infect. Australia; 2018;3(3):156-64.

29. Conterno LO, Turchi MD. Antibiotics for treating chronic osteomyelitis in adults. Cochrane database Syst Rev. England; 2013 Sep;(9):CD004439.

30. Dartnell J, Ramachandran M, Katchburian M. Haematogenous acute and subacute paediatric osteomyelitis: A systematic review of the literature. Bone Joint J. 2012;94-B(5):584-95.

31. Olasinde AA, Oluwadiya KS, Adegbehingbe OO. Treatment of brodie's abscess: Excellent results from curettage, bone grafting and antibiotics. Singapore Med J. 2011 Jun;52(6):436-9. 